\title{
Case report: renal cell carcinoma of first kidney allograft in transplanted recipient for second time
}

\begin{abstract}
The uses of immunosuppressive therapy to preserve the new allograft function can significantly increases the incidence of malignancies in transplanted recipients compared with that of general population. The ability to identify and prevent solid organ tumours in the transplant patients, particularly early stages carcinomas depends on regular screening examinations and strict adherence to prophylactic measures. Screening of the patient and donor prior to transplant can help to detect any underlying pre-existing malignancy. Identification of tumor in renal allograft are usually accidental following ultrasonography, CT scan or MRI imaging, so special attention is essential during any imaging examination of renal allograft to detect an early probable tumours. We hereby, present a case record of 51 years old male who had his first kidney transplant in 1987 on the right side from living unrelated donor; he had approached ESRD in 2006 and underwent his second renal transplantation in June 2006 on the left side from living unrelated donor. Though he had stable kidney function, he started to have intermittent haematuria with lump at right lower loin. Allograft sonography had revealed a big mass in the first right transplanted kidney. CT scan with contrast demonstrated a large lobulated enhancing mass arising from the iliac fossa of first transplanted kidney with local, right common iliac vein metastasis and lungs metastasis. Due to above finding patient underwent right allograft nephrectomy.
\end{abstract}

Volume 3 Issue 6 - 2016

\author{
Hind H AlNour, Fakhriya J Alalawi,Amna \\ Khalifa, Salam Alhasani \\ Department of Nephrology and Urology, Dubai hospital, UAE
}

Correspondence: Hind H AINour, Department of Nephrology, Dubai Health Authority, Dubai, UAE, Tel 0097I50-674620I, Email salamalhasani@hotmail.com

Received: October 29, 2016 | Published: December 30, 2016

Keywords: Immunosuppression; Kidney transplantation, Renal cell carcinoma

Abbreviations: ESRD, end stage renal diseases; NODAT, new onset diabetes after transplant; RCC, renal cell carcinoma; ARCD, acquired renal cystic disease; PCR, polymerase chain reaction; PSA, prostate specific antigen

\section{Introduction}

A post-transplant tumours are variable with large diverse in incidences and prognosis. Malignancies are considered to be the second most common cause of mortality among transplanted recipients after cardiovascular diseases. ${ }^{1}$ In patients with the renal transplant; malignancies of the urinary tract is the second most common following skin tumours. The most affected organs are prostate, kidneys, bladder and testes. ${ }^{2}$ Diagnosis of tumours in renal allograft are usually accidental following ultrasonography, CT scan or MRI imaging, so special attention is essential during imaging examination of renal allograft to detect any probable tumor at early stage. $^{3}$

\section{Case report}

A 51 years old male, with history of end stage renal disease due to chronic glomerulonephritis, had undergone his first kidney transplantation in 1987 at right side from living unrelated donor. He was maintained on three immunosuppressant's; prednisolone cyclosporine and azathioprine. He was doing well with baseline creatinine of $1.2 \mathrm{mg} / \mathrm{dl}$ to $1.5 \mathrm{mg} / \mathrm{dl}$, subsequently his serum creatinine start rising gradually till he approached End Stage Renal Diseases (ESRD) by 2006. A second renal transplantation was done in June 2006 at the left side from living unrelated donor, thereafter he was maintained on steroid, cyclosporine and Mycophenolate with baseline creatinine of $1.2 \mathrm{mgdl}$ to $1.3 \mathrm{mgdl}$, he had stable allograft function. In 2013 he developed new onset diabetes after transplant (NODAT). Due to urethral stricture he underwent several internal urethrotomy procedures in the past few years.

Over a few months, he was complaining of intermittent haematuria with a bulge noticed at the right lower loin, Ultrasound was done in April 2015; and revealed a big mass in the right old transplant kidney (see image 1A, 1B). CT scan with contrast was done (image 2A, 2B), and revealed a large lobulated outlines heterogeneously enhancing mass arising from the right iliac fossa of the transplanted kidney (measures $8.7 \times 6.9 \times 11.5 \mathrm{~cm}$ in diameter) with local invasion, lungs metastasis and right common iliac vein tumor thrombus. Due to above finding patient underwent right allograft nephrectomy, with uneventful post-operative course. Biopsy of the transplanted kidney consists with clear renal cell carcinoma. His immunosuppression had been modulated by stopping Mycophenolate and replacing cyclosporine by Everolimus. He is also under oncology follow up (Figure 1A,1B \& Figure 2A,2B).
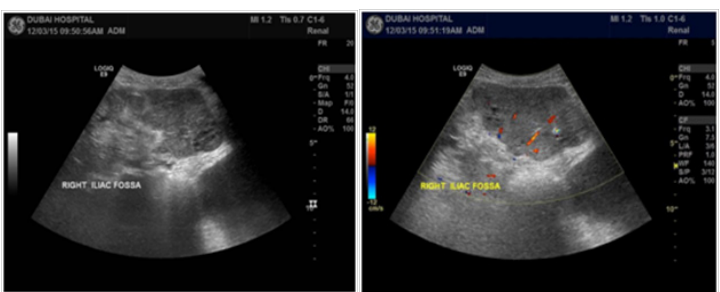

Figure IA \& IB: Old Transplanted kidney in the right iliac fossa shows a large hypoechoeic solid lobulated mass measuring I0.0x6.9cm with increased vascularity on Doppler. 

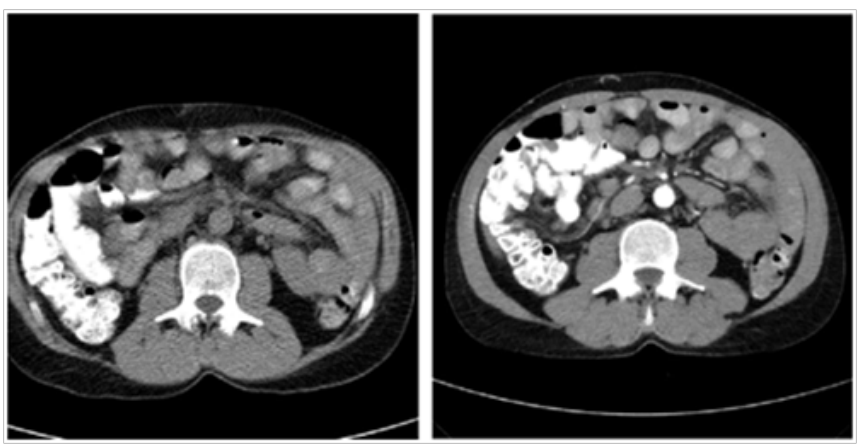

Figure 2A \& 2B: Revealed a large lobulated outlines heterogeneously enhancing mass arising from the iliac fossa of transplanted kidney (measures $8.7 \times 6.9 \times 11.5 \mathrm{~cm}$ in diameter).

\section{Discussion}

Kidney transplant recipients are at higher risk of developing carcinoma of the native kidneys especially if they were on long dialysis period, ${ }^{4,5}$ the exact cause behind these remains unclear; it was postulated that different factors like developing tubular hyperplasia during the duration of advanced kidney failure can be attributed. Alternative cause is cyst formation with likely chance of malignant transformation. ${ }^{4,6}$ Other factors like genetic factors, chronic viral infections, cigarette smoking, radiation, longer graft survival, older donor as well as recipient, African Americans and introduction of more potent immunosuppression were all encountered too. ${ }^{6,7}$ The occurrence of graft rejection in the first post-transplant year can increase the incidence of secondary neoplasm; possibly due to escalation of immunosuppressive level required to treat the rejection episode. ${ }^{4,6}$

\section{Role of immunosuppression in the development of post-transplant malignancies}

The increased risk of post-transplant malignancy is consistent with the increased levels of immunosuppressive therapy. Studies had demonstrated that usage of cyclosporine as well as tacrolimus, may promote malignancy following renal transplantation through an increased production of transforming growth factor beta. ${ }^{4}$ The use of azathioprine has been associated with post-transplant malignancy, particularly an increased risk of cutaneous squamous cell carcinomas. ${ }^{4}$ On the other hand, there is convincing proof that avoiding CNIs and use of mTOR-inhibitors (such as Sirolimus and Everolimus) can convey advantages as for the advancement of malignancy., ${ }^{4,-12}$ this was illustrated on several studies, e.g. patients in CONVERT study who were converted to sirolimus; had a reduction in the rate of malignancies compared to those who remained on CNI at 12 and 24 months (3\% versus 10\%). ${ }^{9,13-15}$ mTOR-I has cytostatic effect and no cytotoxic activities, so the clinical effect is stabilization of the tumor cells rather than progression.

The mechanism by which m-TOR-I can induce this anti-tumor activity is related to up-regulation of adhesion molecules and to a change to less aggressive phenotype of tumoral cells, as shown with Luan et al. ${ }^{16}$ who demonstrated that Sirolimus can modify tumor cells from aggressive phenotypes like spindle- or dome-shaped cells to a less aggressive cuboidal form in renal cell carcinoma. Additionally, m-TORI can block growth-factor-stimulating cell multiplication of haematopoietic and non-haematopoietic cells (e.g. CNS, hepatocytes, renal, melanocytic, osteoblastic, myogenic, fibroblasts and endothelial cells) by forming a complex with the intracellular immunophilins FKBP-12. In the pre-clinical models; m-TORI had demonstrated growth inhibition of tumoral cells by expanding expression of E-cadherin and p27-kip1, additionally it diminish cyclin-d1 expression, causing arrest in cellular progression from $\mathrm{G} 1$ to S-phase. ${ }^{13,14,17-19}$ Conversely, mTOR-I are associated with an increased risk of mortality secondary to cardiovascular disease and infections.

\section{Renal cell carcinoma of the grafted kidney}

Renal cell carcinoma (RCC) is the most frequent malignant kidney tumor. Its incidence in transplanted patients is slightly higher than in the general population (4.8 vs. $2.3 \%$ ). Most of these tumours are situated in the native kidneys, while less than $10 \%$ is located in the allograft. ${ }^{6,20,21}$ Renal cell carcinoma in kidney transplant recipients can originate either from the donor, or as a pre-existent tumor in the recipient before transplantation, or as a de-novo malignancy that occur post-transplant in the native kidney or in allograft. ${ }^{20,21}$ Generally, they have less aggressive nature in transplanted patients, independently of their location, except for the papillary tumor RCC, in which an increased aggressiveness is reported. Papillary renal cell carcinoma represents $5-15 \%$ of overall kidney neoplasm. ${ }^{22,23}$ Moreover, papillary tumours are more frequent in the transplanted population than in the general population and are usually associated with acquired renal cystic disease (ARCD) of the native kidneys rather that allograft. ${ }^{20}$

Absence of graft innervation had provoked those patients to be asymptomatic at the time of diagnosis, and usually they are discovered incidentally when routine imaging is carried out during the periodic follow-up. Therefore it is recommended to monitor post-transplant recipients for microscopic or gross haematuria by urine analysis every three to six-months; if this is positive then urine culture has to be done in addition to urine cytology, furthermore urine polymerase chain reaction (PCR) for BK polyomavirus, prostate specific antigen (PSA) in male patients, and ultrasound of the native kidney and transplanted kidney with the bladder should be done. ${ }^{4,20}$ Additionally, it has been advised to perform routine ultrasonography of the transplanted kidney lifelong on yearly basis. ${ }^{3}$ This technique allows initial diagnosis to be made, whereas the images can be confirmed by CT or MRI thereafter. ${ }^{20}$

\section{Treatment}

Radical nephrectomy and radiotherapy can cure patients with renal cell carcinoma. Radical nephrectomy were justified by a large lesion, a low functioning graft, two or more lesions, locally invasive tumor or diffuse graft infiltration on radiological studies. ${ }^{2}$ In the recent years, partial nephrectomy (Nephron sparing surgery) has been performed widely with great success. It is usually performed when the tumours are clinically localized and the size of the tumor is $6 \mathrm{~cm}$ or less (Stage 1A and possibly stage 1B). ${ }^{2,21,24,25}$ Percutaneous radiofrequency or cryoablation therapy is well-established treatment for small and exophytic lesions since it is less invasive and has a low mortality rate besides it avoids renal ischemia associated with vascular clamping which is occasionally necessary. ${ }^{23}$ Minimization or withdrawal of Calcineurin inhibitors and replace it with mTOR inhibitor (Sirolimus or Everolimus) is recommended..$^{23}$ However, some authors prefer to maintain similar immunosuppressive schemes without modifications, as this will lead to decrease in the rejection risk and hence no clear correlation between immunosuppression and tumours in some reported literatures. ${ }^{26}$ 


\section{Conclusions and recommendations}

The ability to identify and prevent solid organ tumours in the transplant recipients, especially early stage carcinomas depends on regular screening examinations and strict adherence to prophylactic measures.

I. Cautious screening of the patient and donor prior to transplantation can help in detecting any underlying pre-existing malignancy. ${ }^{3}$

II. Immunologic human leukocyte antigen typing and (DNA) genetic analysis should be done on each tumor specimen when possible, to detect the recipient or donor origin of the malignancy. $3,4,23,27-29$

III. Renal cell carcinoma in post-transplant recipients is commonly to occur in the native kidneys rather than allograft kidney.

IV. Renal allograft sonography should be done annually for life. ${ }^{2}$

$\mathrm{V}$. When the tumor is symptomatic, it often has a poorer prognosis. ${ }^{23}$

VI. If the tumor is small and localized; it can be treated with conservative management like radio frequency ablation or partial nephrectomy.

VII. Total transplant nephrectomy can give a durable cure but it will return patient to chronic dialysis.

\section{Acknowledgments}

None.

\section{Conflicts of interest}

The author declares there is no conflict of interest.

\section{References}

1. Végsö G, Toronyi E, Hajdu M, et al. Renal cell carcinoma of the native kidney: a frequent tumor after kidney transplantation with favorable prognosis in case of early diagnosis. Transplant Proc. 2011;43(4):12611263.

2. Ianhez LE, Lucon M, Nahas WC, et al. Renal Cell Carcinoma in Renal Transplant Patients. Urology. 2007;69(3):462-464.

3. Leonardou P, Semelka RC, Mastropasqua M, et al. Renal cell carcinoma in a transplanted kidney: MR imaging findings. Magn Reson Imaging. 2003;21(6):691-693.

4. Brennan DC, Rodeheffer RJ, Ambinder RF, et al. Development of malignancy following solid organ transplantation. 2015.

5. Birkeland SA, Løkkegaard H, Storm HH. Cancer risk in patients on dialysis and after renal transplantation, Research letters. THE LANCET. 2000;355(9218):1886-1887.

6. Hurst FP, Jindal RM, Graham LJ, et al. Incidence, Predictors, Costs, and Outcome of Renal Cell Carcinoma After Kidney Transplantation: USRDS Experience. Transplantation. 2010;90(8):898-904.

7. Chapman JR, Webster AC, Wong G. Cancer in the transplant recipient Cold Spring Harb Perspect Med. 2013;3(7):a015677.

8. Russ GR. Optimising the use of mTOR inhibitors in renal Transplantation. Transplantation Res. 2013;2(Suppl 1):S4.

9. Flavio Vincenti. CNI Sparing With mTOR Inhibitors in Kidney Transplantation. Medscape Education Transplantation. 2012.

10. Johnson RW, Kreis H, Oberbauer R, et al. Sirolimus Allows Early Cyclosporine Withdrawal In Renal Transplantation Resulting In
Improved Renal Function And Lower Blood Pressure. Transplantation. 2011;72(5):777-786

11. Oberbauer R, Segolini G, Campistol JM, et al. Early cyclosporine withdrawal from a sirolimus-based regimen results in better renal allograft survival and renal function at 48 months after transplantation. Transplant Int. 2005;18(1):22-28.

12. Piselli P, Busnach G, Citterio F, et al. Kidney transplant and cancer risk: an epidemiological study in Northern and Central Italy. Epidemiol Prev. 2008;32(4-5):205-211.

13. Morath C, Arns W, Schwenger V, et al. Sirolimus in renal transplantation. Nephrol Dial Transplant. 2007;22(Suppl 8):viii61-viii65.

14. Patel SJ, Dawson KL, Knight RJ, et al. The Role of mTOR Inhibition in Renal Transplant Immune Suppression. Dialysis \& Transplantation. 2011;40(1).

15. Schena FP, Pascoe MD, Alberu J, et al. Conversion From Calcineurin Inhibitors to Sirolimus Maintenance Therapy in Renal Allograft Recipients: 24-Month Efficacy and Safety Results From the CONVERT Trial. Transplantation. 2009;87(2):233-242.

16. Luan FL, Hojo M, Maluccio M, et al. Rapamycin blocks tumor progression: unlinking immunosuppression from antitumor efficacy. Transplantation. 2002;73(10):1565-1572.

17. Gianluigi Zaza, Paola Tomei, Paolo Ria, et al. Systemic and Nonrenal Adverse Effects Occurring in Renal Transplant Patients Treated with mTOR Inhibitors. Clinical and Developmental Immunology. 2013;Article ID403280.

18. Nashan B. Maximizing the clinical outcome with $m$ TOR inhibitors in the renal transplant recipient: defining the role of calcineurin inhibitors. Transpl Int. 2004;17(6):179-285.

19. Nguyen C, Shapiro R. New immunosuppressive agents in pediatric transplantation. Clinics (Sao Paulo). 2014;69(S1):8-16.

20. González-López R, Bueno-Serrano G, Mayor-De Castro J, et al. Clinical presentation of renal cell carcinoma in renal transplant. Arch Esp Urol. 2009;62(3):207-213.

21. Penn I. Primary kidney tumors before and after transplantation Transplantation. 1995;59(4):480-485.

22. Tsui KH, van Ophoven A, Shvarts O, et al. Nephron-Sparing Surgery for Renal Cell Carcinoma. Rev Urol. 1999;1(4):216-225.

23. Tillou X, Guleryuz K, Collon S, et al. Renal cell carcinoma in functional renal graft: Toward ablative treatments. Transplant Rev (Orlando). 2016;30(1):20-26

24. Ghafari A. Transplantation of a Kidney with a Renal Cell Carcinoma after Living Donation: a Case Report. Transplant Proc. 2007;39(5):16601661.

25. Siebels M, Theodorakis J, Liedl B, et al. Large de novo renal cell carcinoma in a 10-year-old transplanted kidney: Successful OrganPreserving Therapy. Transplantation. 2000;69(4):677-679.

26. Moudoni SM, Lakmichi A, Tligui M, et al. Renal cell carcinoma of native kidney in renal transplant recipients. BJU Int. 2006;98(2):298302

27. Cornelis F, Buy X, André M, et al. De Novo Renal Tumors Arising in Kidney Transplants: Midterm Outcome after Percutaneous Thermal Ablation. Radiology. 260(3):900-907.

28. Kapoor A. Malignancy in Kidney Transplant Recipients. Drugs 2011;68(1):11-19.

29. Rouprêt M, Peraldi MN, Thaunat O, et al. Renal cell carcinoma of the grafted kidney: how to improve screening and graft tracking. Transplantation. 2004;77(1):146-148. 